\title{
Scattering of flexural waves from a hole in a thin plate with an internal beam
}

\author{
A. Climente ${ }^{\mathrm{a})}$ \\ Wave Phenomena Group, Department of Electronic Engineering, Universitat Politècnica de València, \\ Camino de Vera s.n. (Edificio 7 F), ES-46022 Valencia, Spain \\ Andrew N. Norris \\ Mechanical and Aerospace Engineering, Rutgers University, Piscataway, New Jersey 08854 \\ José Sánchez-Dehesa \\ Wave Phenomena Group, Department of Electronic Engineering, Universitat Politècnica de València, \\ Camino de Vera s.n. (Edificio 7 F), ES-46022 Valencia, Spain
}

(Received 7 July 2014; revised 17 November 2014; accepted 25 November 2014)

\begin{abstract}
The scattering of flexural waves by a hole in a thin plate traversed by a beam is modeled here by coupling the Kirchhoff-Love and the Euler-Bernoulli theories. A closed form expression is obtained for the transfer matrix (T-matrix) relating the incident wave to the scattered cylindrical waves. For this purpose, a general method has been developed, based on an analogous impedance method for acoustic waves, for calculating the T-matrix for flexural wave scattering problems. The T-matrix for the problem considered displays a simple structure, composed of distinct sub-matrices which decouple the inside and the outside fields. The conservation of energy principle and numerical comparisons with a commercial finite element simulator have been used to prove the theory.
\end{abstract}

(C) 2015 Acoustical Society of America. [http://dx.doi.org/10.1121/1.4904551]

[OU]

Pages: 293-302

\section{INTRODUCTION}

Propagation of flexural waves in thin plates has been a longstanding topic in the study of elastic materials. Recently, it is receiving increasing attention due to the possibilities in their control offered by new structures like phononic crystals, ${ }^{1,2}$ with applications such as positive ${ }^{3,4}$ and negative refractive lenses. ${ }^{5-7}$ Moreover, by introducing anisotropy in the system, more complicated structures were designed in order to obtain novel elastic devices ${ }^{8,9}$ or others based on thickness variation of the plate. ${ }^{10-13}$

In addition to the phenomena described above, it was demonstrated that by embedding local resonances in a thin plate, the elastic properties can be adjusted to open band gaps and modifying effectively the propagation of flexural waves. ${ }^{14,15}$ Usually the band gaps originate from the periodicity of the structure, falling into the wavelength region on the order of the structural period, but in this case, the band gap position is associated with the frequency of the resonance. This method opens the door to the possibility of studying new devices for controlling flexural wave propagation.

The local resonances have been achieved by introducing surface "inclusions" on top of semi-infinite mediums ${ }^{16}$ or thin plates, such as hollow spheres or cylinders, ${ }^{17}$ springmasses, ${ }^{18,19}$ simple pillars, ${ }^{20}$ or composite pillars. ${ }^{21}$ Experiments have been reported proving the existence of tunable band gaps by using stubs ${ }^{22,23}$ or piezoelectric shunts ${ }^{24}$ on plates. Examples of potential applications of this local resonator, range from vibration absorption ${ }^{25}$ to an elastic analogous of graphene. ${ }^{26}$ For more

\footnotetext{
a) Author to whom correspondence should be addressed. Electronic mail: alclial0@gmail.com
}

information on this topic, the reader is referred to the reviews ${ }^{27-29}$ and references therein.

This work presents a theory that models resonators obtained by cutting out cylindrical segments of a thin plate. The method can be easily generalized to more complicated structures with any combination of connected cylindrical plates with radial beams, such as the resoator described in Ref. 30 . The present method is focused on generating the transfer matrix (T-matrix), which relates arbitrary incident waves to scattered waves. While it is straightforward to derive the diagonal Tmatrix for homogeneous cylindrical targets, ${ }^{31}$ and the computation of the non-diagonal T-matrix for rigid inclusions and holes of arbitrary shape can be performed by quadrature, ${ }^{32}$ the present case is more complicated. The T-matrix is not diagonal and it depends crucially upon the internal structure. This issue is addressed using a flexural wave formulation of the impedance matrix method ${ }^{33}$ proposed for acoustics.

The paper is structured as follows: An introduction to the main problem, the equations used and the boundary conditions are given in Sec. II. The impedance method used to obtain the flexural T-matrix of the system is described in Sec. III. Section IV summarizes the results that are used in Sec. V, where numerical simulations are presented that confirm the solution and examine its properties. Conclusions are presented in Sec. VI. Finally, the Appendixes provides an extended explanation of some steps and results required for the general solution.

\section{PROBLEM DEFINITION}

Figure 1 shows a three dimensional (3D) scheme (left view) and a two dimensional (2D) scheme (right view) of the system analyzed in this work. A flexural wave with linear wavefront is incident in an arbitrary direction on a hole 


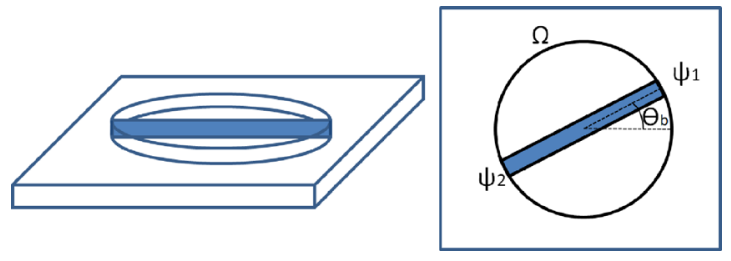

FIG. 1. (Color online) 3D Scheme (left) and 2D scheme (right) of a thin plate with a hole (cylindrical boundary $\Omega$ ) traversed by a beam (oriented at angle of $\left.\theta_{b}\right)$ connected to the plate at two anchor points $\left(\Psi_{i} ; i=1,2\right)$.

(cylindrical boundary $\Omega$ ) in the thin plate. Inside, there is a beam connected to the plate at two anchor points $\left(\Psi_{i} ; i=1\right.$, 2). The beam is inclined at an angle of $\theta_{b}$ and is made of the same material as the plate. The problem can be easily generalized to the case of different materials as the equations are easily coupled. The motion of the flexural wave $W$ interacts with the motion of the beam $V$ and creates a scattered wave. Time harmonic solutions of the form $e^{-i \omega t}$ are assumed throughout, where $\omega$ is the angular frequency.

\section{A. Flexural wave solution in the plate}

The equation of motion of the flexural wave is given by the Kirchhoff-Love theory for thin plates as

$$
\nabla^{4} W-k_{p}^{4} W=0
$$

where

$$
\nabla=\frac{\partial}{\partial r} \hat{r}+\frac{1}{r} \frac{\partial}{\partial \theta} \hat{\theta}
$$

in polar coordinates. Additionally, $W$ is the displacement in the $\hat{z}$-direction, $k_{p}=\left(\rho h \omega^{2} / D\right)^{1 / 4}$ is the wavenumber, $h$ is the thickness of the plate, $D=E h^{3} / 12\left(1-\nu^{2}\right)$ is the flexural stiffness, $E$ is the Young's modulus, $\rho$ is the mass density, and $\nu$ is the Poisson's ratio. The displacement $W$ can be expressed as an infinite sum of Bessel, $J_{q}(x)$, Hankel, $H_{q}(x)\left[\equiv H_{q}^{(1)}(x)\right]$, and modified Bessel functions, $I_{q}(x)$ and $K_{q}(x)$, as

$$
W=\sum_{q} W_{q}(r) e^{i q \theta}=\sum_{q}\left[W_{q}^{\text {inc }}(r)+W_{q}^{\text {scat }}(r)\right] e^{i q \theta}=\sum_{q}\left[A_{q}^{(J)} J_{q}\left(k_{p} r\right)+A_{q}^{(I)} I_{q}\left(k_{p} r\right)+B_{q}^{(H)} H_{q}\left(k_{p} r\right)+B_{q}^{(K)} K_{q}\left(k_{p} r\right)\right] e^{i q \theta}
$$

where $A_{q}^{(J)}$ and $A_{q}^{(I)}$ are the incoming wave coefficients and $B_{q}^{(H)}$ and $B_{q}^{(K)}$ are the scattered wave coefficients. The index $q$ ranges from $-\infty$ to $+\infty$. Let us define two column vectors $\hat{A}_{q}=\left[A_{q}^{(J)} A_{q}^{(I)}\right]^{t}$ and $\hat{B}_{q}=\left[B_{q}^{(H)} B_{q}^{(K)}\right]^{t}$.

The four boundary conditions for a thin plate are the continuity of the displacement $W$, the slope $W_{r}^{\prime}=\partial W / \partial r$, the radial moment $M_{r}$ and the radial Kirchhoff stress $V_{r}$, where

$$
\begin{aligned}
& M_{r}=-D\left[\frac{\partial^{2} W}{\partial r^{2}}+\nu\left(\frac{1}{r} \frac{\partial W}{\partial r}+\frac{1}{r^{2}} \frac{\partial^{2} W}{\partial \theta^{2}}\right)\right] \\
& V_{r}=-D\left[\frac{\partial}{\partial r} \Delta W+(1-\nu) \frac{1}{r^{2}} \frac{\partial}{\partial \theta}\left(\frac{\partial^{2} W}{\partial r \partial \theta}-\frac{1}{r} \frac{\partial W}{\partial \theta}\right)\right] .
\end{aligned}
$$

\section{B. Beam solution}

Inside the hole is a rectangular section beam with length $2 R$, height $h$, and width $b$. The equation of motion of the beam is

$$
\frac{\partial^{4} V}{\partial x^{4}}-k_{b}^{4} V=0
$$

where $V$ is the displacement in the $\hat{z}$-axis, $k_{b}=\left(m \omega^{2} / \mathrm{EI}\right)^{1 / 4}$ is the wave number, $E$ is the Young's modulus, $I=b h^{3} / 12$ is the second moment of area, $m=b h \rho$ is the mass per unit length of the beam, and $\rho$ is the mass density. The displacement $V$ can be expressed

$$
V(x)=C^{I} e^{+i k_{b} x}+C^{I I} e^{-i k_{b} x}+D^{I} e^{+k_{b} x}+D^{I I} e^{-k_{b} x},
$$

where $C^{I}$ and $C^{I I}$ are the coefficients of the forward traveling waves and $D^{I}$ and $D^{I I}$ are the coefficients of the backward traveling waves.

The four boundary conditions for a beam are the continuity of the displacement $V$, the slope $V_{r}^{\prime}=\partial V / \partial r$, the moment $M_{x}$, and the shear stress $Q_{x}$,

$$
\begin{aligned}
& M_{x}=-E I \frac{\partial^{2} V}{\partial x^{2}} \\
& Q_{x}=\frac{\partial M_{x}}{\partial x}=-E I \frac{\partial^{3} V}{\partial x^{3}} .
\end{aligned}
$$

\section{Coupling boundary conditions}

The boundary conditions for a hole in a thin plate are $M_{r}=0$ and $V_{r}=0$ at the boundary $\Omega$. For the problem considered, the beam introduces additional conditions at the anchor points in $\Psi_{i}$ requiring that the displacement and the slope are continuous and the sum of the moments and the sum of the stresses are zero. See Appendix A for more information. In summary, the boundary conditions are the following:

$$
\begin{aligned}
& W(R, 0)=V(R) \quad \text { and } \quad W(R, \pi)=V(-R), \\
& \left.\frac{\partial W}{\partial r}\right|_{\substack{r=R \\
\theta=0}}=\left.\frac{\partial V}{\partial x}\right|_{x=R} \text { and }\left.\frac{\partial W}{\partial r}\right|_{\substack{r=R \\
\theta=0}}=-\left.\frac{\partial V}{\partial x}\right|_{x=-R},
\end{aligned}
$$




$$
\begin{aligned}
& \left.M_{r}\right|_{\substack{r=R \\
\theta=0}}=\left.\frac{M_{x}}{R}\right|_{x=R} \text { and }\left.\quad M_{r}\right|_{\substack{r=R \\
\theta=\pi}}=\left.\frac{M_{x}}{R}\right|_{x=-R}, \\
& \left.V_{r}\right|_{\substack{r=R \\
\theta=0}}=\left.\frac{Q_{x}}{R}\right|_{x=R} \text { and }\left.\quad V_{r}\right|_{\substack{r=R \\
\theta=\pi}}=-\left.\frac{Q_{x}}{R}\right|_{x=-R} .
\end{aligned}
$$

\section{T-MATRIX SOLUTION}

This section develops the explicit solution for the Tmatrix that relates the incoming and the scattered coefficients as $\hat{B}=\mathbf{T} \hat{A}$. The T-matrix is central to describing scattering solutions as it allows us to express the scattered field for any type of incident wave. Additionally, we will obtain the coefficients $\left(C^{I}, C^{I I}\right)$ and $\left(D^{I}, D^{I I}\right)$ that describe the wave motion on the internal beam.

\section{A. Impedance method for plates}

In order to couple the dynamics of the external plate with the internal beam, we develop here a method for flexural waves that is analogous to the one described for acoustic waves in Ref. 33. In this work, the author provides a method of obtaining the T-matrix of the fluid-fluid interface as the combination of three impedance matrices. Two of them describe the background and the third one describes the internal media. In our case (see Appendix B for more information),

$$
\begin{aligned}
\mathbf{T}_{q s}= & {\left[\mathbf{M}_{q q}^{H K}\left(k_{p}\right)\right]^{-1}\left[\mathbf{Z}_{q s}^{\text {scat }}+\mathbf{Z}_{q s}\right]^{-1} } \\
& \times\left[\mathbf{Z}_{q s}^{\text {inc }}-\mathbf{Z}_{q s}\right]\left[\mathbf{M}_{s s}^{J I}\left(k_{p}\right)\right],
\end{aligned}
$$

where the three impedance matrices are defined by

$$
\left[\begin{array}{c}
M_{r} \\
V_{r}
\end{array}\right]_{q}^{(\mathrm{inc})}=-\mathbf{Z}_{q s}^{(\mathrm{inc})}\left[\begin{array}{c}
W \\
W_{r}^{\prime}
\end{array}\right]_{s}^{(\mathrm{inc})} \text { on } \Omega,
$$

$$
\begin{aligned}
& {\left[\begin{array}{c}
M_{r} \\
V_{r}
\end{array}\right]_{q}^{\text {(scat) }}=\mathbf{Z}_{q s}^{\text {(scat) }}\left[\begin{array}{c}
W \\
W_{r}^{\prime}
\end{array}\right]_{s}^{\text {(scat) }} \text { on } \Omega,} \\
& \left(\left[\begin{array}{c}
M_{r} \\
V_{r}
\end{array}\right]_{q}^{\text {(inc) }}+\left[\begin{array}{c}
M_{r} \\
V_{r}
\end{array}\right]_{q}^{\text {(scat) }}\right) \\
& \quad=-\mathbf{Z}_{q s}\left(\left[\begin{array}{c}
W \\
W_{r}^{\prime}
\end{array}\right]_{s}^{\text {(inc) }}+\left[\begin{array}{c}
W \\
W_{r}^{\prime}
\end{array}\right]_{s}^{\text {(scat) }}\right) \text { on } \Omega,
\end{aligned}
$$

and the two additional matrices appearing in Eq. (8) follow from

$$
\mathbf{M}_{q q}^{\Upsilon \Phi}\left(k_{i}\right)=\left[\begin{array}{cc}
\Upsilon_{q}\left(k_{i} R\right) & \Phi_{q}\left(k_{i} R\right) \\
k_{i} \Upsilon_{q}^{\prime}\left(k_{i} R\right) & k_{i} \Phi_{q}^{\prime}\left(k_{i} R\right)
\end{array}\right],
$$

where $k_{i}$ is a dummy variable. For example, $k_{i}=k_{p}$ in Eq. (8).

\section{B. Background impedances}

Based on the definitions of Eqs. (9a) and (9b), the background impedances are obtained as

$$
\begin{aligned}
& \mathbf{Z}_{q q}^{(i n c)}=-\mathbf{N}_{q q}^{J I}\left(k_{p}\right)\left[\mathbf{M}_{q q}^{J I}\left(k_{p}\right)\right]^{-1}, \\
& \mathbf{Z}_{q q}^{(s c a t)}=\mathbf{N}_{q q}^{H K}\left(k_{p}\right)\left[\mathbf{M}_{q q}^{H K}\left(k_{p}\right)\right]^{-1},
\end{aligned}
$$

where

$$
\mathbf{N}_{q q}^{\Upsilon \Phi}\left(k_{i}\right)=\left[\begin{array}{cc}
S_{q}^{\Upsilon}\left(k_{i}\right) & S_{q}^{\Phi}\left(k_{i}\right) \\
T_{q}^{\Upsilon}\left(k_{i}\right) & T_{q}^{\Phi}\left(k_{i}\right)
\end{array}\right] .
$$

The following notation applies:

$$
\begin{aligned}
& S_{q}^{\xi}\left(k_{i}\right)=-\frac{D}{R^{2}}\left\{\left[q^{2}(1-\nu) \mp\left(k_{i} R\right)^{2}\right] \xi_{q}\left(k_{i} R\right)-[(1-\nu)]\left(k_{i} R\right) \xi_{q}^{\prime}\left(k_{i} R\right)\right\}, \\
& T_{q}^{\xi}\left(k_{i}\right)=-\frac{D}{R^{3}}\left\{\left[q^{2}(1-\nu)\right] \xi_{q}\left(k_{i} R\right)-\left[q^{2}(1-\nu) \pm\left(k_{i} R\right)^{2}\right]\left(k_{i} R\right) \xi_{q}^{\prime}\left(k_{i} R\right)\right\},
\end{aligned}
$$

where the upper signs are used for $\xi=J, H$ and the lower signs for $\xi=I, K$. To simplify $S_{q}^{\xi}\left(k_{i}\right)$ and $T_{q}^{\xi}\left(k_{i}\right)$, the second order ordinary differential equations of these functions and the Wronskian identities $J_{q}(x) H_{q}^{\prime}(x)-J_{q}^{\prime}(x) H_{q}(x)=2 i / \pi x$ and $I_{q}(x) K_{q}^{\prime}(x)-I_{q}^{\prime}(x) K_{q}(x)=-(1 / x)$ have been used.

\section{Internal impedance}

The third impedance matrix required for the solution of Eq. (8), which is associated with the internal beam, is derived here. On the circular boundary we have $\Omega,\left(M_{r}=0, V_{r}=0\right)$ $\forall \theta$ except for $(\theta=0, \theta=\pi)$, hence the two boundary conditions $(7 \mathrm{c})$ and $(7 \mathrm{~d})$ can be rewritten

$$
\begin{aligned}
\left.M_{r}(\theta)\right|_{r=R} & \equiv M_{x}(\theta) \\
& =\frac{1}{R}\left[M_{x}(R) \delta\left(\theta-\theta_{\Psi_{1}}\right)+M_{x}(-R) \delta\left(\theta-\theta_{\Psi_{2}}\right)\right],
\end{aligned}
$$




$$
\begin{aligned}
\left.V_{r}(\theta)\right|_{r=R} & \equiv Q_{x}(\theta) \\
& =\frac{1}{R}\left[Q_{x}(R) \delta\left(\theta-\theta_{\Psi_{1}}\right)-Q_{x}(-R) \delta\left(\theta-\theta_{\Psi_{2}}\right)\right] .
\end{aligned}
$$

Knowing that $\theta_{\Psi_{1}}=\theta_{b}$ and $\theta_{\Psi_{2}}=\theta_{b}+\pi$ and expanding Eqs. (14a) and (14b) in azimuthal orders, using $\delta\left(\theta-\theta_{i}\right)=(1 / 2 \pi) \sum_{q} e^{i q\left(\theta-\theta_{i}\right)}$, we obtain

$$
\begin{aligned}
M_{x}(\theta) & =\sum_{q} M_{q} e^{i q \theta} \\
& =\frac{1}{2 \pi R} \sum_{q}\left[M_{x}(R)+(-1)^{q} M_{x}(-R)\right] e^{i q\left(\theta-\theta_{b}\right)}, \\
Q_{x}(\theta) & =\sum_{q} Q_{q} e^{i q \theta} \\
& =\frac{1}{2 \pi R} \sum_{q}\left[Q_{x}(R)-(-1)^{q} Q_{x}(-R)\right] e^{i q\left(\theta-\theta_{b}\right)} .
\end{aligned}
$$

These equations can be combined and rewritten in matrix form as

$$
\left[\begin{array}{c}
M_{x} \\
Q_{x}
\end{array}\right]_{q}=\frac{e^{-i q \theta_{b}}}{2 \pi R}\left(\left[\begin{array}{c}
M_{R} \\
Q_{R}
\end{array}\right]+(-1)^{q}\left[\begin{array}{c}
M_{-R} \\
-Q_{-R}
\end{array}\right]\right) .
$$

Following the steps explained in Appendix C, we obtain the $4 \times 4$ stiffness matrix $\mathbf{K}$ that relates the displacement and the slope at the two ends of a beam with the moments and shear stresses, as

$$
\left[\begin{array}{c}
M_{-R} \\
-Q_{-R} \\
M_{R} \\
Q_{R}
\end{array}\right]=\mathbf{K}\left[\begin{array}{c}
V_{-R} \\
-V_{-R}^{\prime} \\
V_{R} \\
V_{R}^{\prime}
\end{array}\right]=\left[\begin{array}{ll}
\mathbf{K}_{1} & \mathbf{K}_{2} \\
\mathbf{K}_{2} & \mathbf{K}_{1}
\end{array}\right]\left[\begin{array}{c}
V_{-R} \\
-V_{-R}^{\prime} \\
V_{R} \\
V_{R}^{\prime}
\end{array}\right]
$$

where the stiffness matrix $\mathbf{K}$ is defined as

$$
\mathbf{K}=\frac{-E I k_{b}^{2}}{1-c c_{h}}\left[\begin{array}{cc|cc}
-s s_{h} & k_{b}^{-1}\left(s c_{h}-c s_{h}\right) & c_{h}-c & -k_{b}^{-1}\left(s_{h}-s\right) \\
-k_{b}\left(c s_{h}+s c_{h}\right) & s s_{h} & k_{b}\left(s+s_{h}\right) & c-c_{h} \\
\hline c_{h}-c & -k_{b}^{-1}\left(s_{h}-s\right) & -s s_{h} & k_{b}^{-1}\left(s c_{h}-c s_{h}\right) \\
k_{b}\left(s+s_{h}\right) & c-c_{h} & -k_{b}\left(c s_{h}+s c_{h}\right) & s s_{h}
\end{array}\right]
$$

with the abbreviated notation $c=\cos \left(2 k_{b} R\right), s=\sin \left(2 k_{b} R\right)$, $c_{h}=\cosh \left(2 k_{b} R\right)$, and $s_{h}=\sinh \left(2 k_{b} R\right)$. For the purpose of this work it is useful to rewrite Eq. (17) as two separate relations,

$$
\begin{aligned}
& {\left[\begin{array}{c}
M_{-R} \\
-Q_{-R}
\end{array}\right]=\mathbf{K}_{1}\left[\begin{array}{c}
V_{-R} \\
-V_{-R}^{\prime}
\end{array}\right]+\mathbf{K}_{2}\left[\begin{array}{c}
V_{R} \\
V_{R}^{\prime}
\end{array}\right],} \\
& {\left[\begin{array}{c}
M_{R} \\
Q_{R}
\end{array}\right]=\mathbf{K}_{2}\left[\begin{array}{c}
V_{-R} \\
-V_{-R}^{\prime}
\end{array}\right]+\mathbf{K}_{1}\left[\begin{array}{c}
V_{R} \\
V_{R}^{\prime}
\end{array}\right] .}
\end{aligned}
$$

Combining Eqs. (16) and (19) yields the following result:

$$
\begin{aligned}
{\left[\begin{array}{c}
M_{x} \\
Q_{x}
\end{array}\right]_{q}=} & \frac{e^{-i q \theta_{b}}}{2 \pi R}\left[\mathbf{K}_{1}+(-1)^{q} \mathbf{K}_{2}\right] \\
& \times\left(\left[\begin{array}{c}
V_{R} \\
V_{R}^{\prime}
\end{array}\right]+(-1)^{q}\left[\begin{array}{c}
V_{-R} \\
-V_{-R}^{\prime}
\end{array}\right]\right) .
\end{aligned}
$$

The two remaining boundary conditions (7a) and (7b) may be written in terms of Fourier coefficients as

$$
\left[\begin{array}{c}
V_{R} \\
V_{R}^{\prime}
\end{array}\right]=\sum_{s}\left[\begin{array}{l}
W_{R} \\
W_{R}^{\prime}
\end{array}\right]_{s} e^{i s \theta_{b}},\left[\begin{array}{c}
V_{-R} \\
-V_{-R}^{\prime}
\end{array}\right]=\sum_{s}(-1)^{s}\left[\begin{array}{l}
W_{R} \\
W_{R}^{\prime}
\end{array}\right]_{s} e^{i s \theta_{b}} .
$$

Combining these boundary conditions with Eq. (20) we obtain

$$
\left[\begin{array}{c}
M_{r} \\
V_{r}
\end{array}\right]_{q}=-\sum_{s} \mathbf{Z}_{q s}\left[\begin{array}{c}
W_{R} \\
W_{R}^{\prime}
\end{array}\right]_{s}
$$

where the $2 \times 2$ blocks of the internal impedance matrix are given by

$$
\mathbf{Z}_{q s}=-\frac{e^{i(s-q) \theta_{b}}}{2 \pi R}\left[1+(-1)^{q+s}\right]\left[\mathbf{K}_{1}+(-1)^{q} \mathbf{K}_{2}\right] .
$$

This concludes the derivation of the required impedance matrices. The full solution is summarized next.

\section{SCATTERING AND INTERNAL COEFFICIENTS}

The results of the Secs. II and III are summarized here. This provides in one place simple formulas for the coefficients of the incoming, the scattered and the beam solution.

\section{A. Incident wave coefficients}

While the general solution can handle arbitrary incidence, here we focus on plane wave or a point source, which will be used in the numerical examples later. The coefficients of an incoming wave for these two possible cases are as follows: 
$\hat{A}_{q}=\left[\begin{array}{c}i^{q} \\ 0\end{array}\right] e^{-i q \theta_{s}}$ for a plane wave in direction $\theta_{s}$,

$A_{q}=\left[\begin{array}{c}H_{q}\left(k_{p} R_{s}\right) \\ \frac{2 i}{\pi} K_{q}\left(k_{p} R_{s}\right)\end{array}\right] e^{-i q \theta_{s}}$ for a point source at $\left(R_{s}, \theta_{s}\right)$.

\section{B. Scattered coefficients}

The coefficients defining the scattered wave are obtained from $\hat{B}_{q}=\mathbf{T}_{q s} \hat{A}_{s}$, where the T-matrix has $2 \times 2$ block elements

$$
\begin{aligned}
\mathbf{T}_{q s}= & {\left[\mathbf{M}_{q q}^{H K}\left(k_{p}\right)\right]^{-1} \quad\left[\mathbf{Z}_{q s}^{\text {scat }}+\mathbf{Z}_{q s}\right]^{-1} } \\
& \times\left[\mathbf{Z}_{q s}^{\text {inc }}-\mathbf{Z}_{q s}\right] \quad\left[\mathbf{M}_{s s}^{J I}\left(k_{p}\right)\right]
\end{aligned}
$$

with

$$
\begin{aligned}
& \mathbf{Z}_{q q}^{(\text {inc })}=-\mathbf{N}_{q q}^{J I}\left(k_{p}\right)\left[\mathbf{M}_{q q}^{J I}\left(k_{p}\right)\right]^{-1}, \\
& \mathbf{Z}_{q q}^{\text {(scat })}=\mathbf{N}_{q q}^{H K}\left(k_{p}\right)\left[\mathbf{M}_{q q}^{H K}\left(k_{p}\right)\right]^{-1}, \\
& \mathbf{Z}_{q s}=-\frac{e^{i(s-q) \theta_{b}}}{2 \pi R}\left(1+(-1)^{q+s}\right)\left[\mathbf{K}_{1}+(-1)^{q} \mathbf{K}_{2}\right],
\end{aligned}
$$

where Eqs. (10), (12), and (18) were used in simplifying terms.

\section{Beam coefficients}

The coefficients for the flexural waves in the internal beam are, see Eq. (5),

$$
\begin{aligned}
& {\left[\begin{array}{c}
C^{I} \\
C^{I I} \\
D^{I} \\
D^{I I}
\end{array}\right]=\left[\mathbf{L}_{1}^{\prime}\right]^{-1} \sum_{q}\left[\begin{array}{cc}
(-1)^{q} \mathbf{M}_{q q}^{J I}\left(k_{p}\right) & (-1)^{q} \mathbf{M}_{q q}^{H K}\left(k_{p}\right) \\
\mathbf{M}_{q q}^{J I}\left(k_{p}\right) & \mathbf{M}_{q q}^{H K}\left(k_{p}\right)
\end{array}\right] } \\
& \times\left[\begin{array}{c}
A_{q}^{(J)} \\
A_{q}^{(I)} \\
B_{q}^{(H)} \\
B_{q}^{(K)}
\end{array}\right] e^{i q \theta_{b}} .
\end{aligned}
$$

These follow from Eqs. (10), (C4), and other results described in Appendix C.

\section{NUMERICAL SIMULATIONS}

In this section the theory developed previously is tested to prove its efficiency. Conservation of energy is used as one measure of the accuracy of the method in predicting the farfield produced over a wide bandwidth. The results are also compared against a commercial finite element method (FEM) simulator.

\section{A. Energy flux}

The principle of conservation of energy has to be met, so if the system is not capable of absorbing or creating waves, the result of calculating the energy flux crossing a circle around the system has to be zero. The energy conservation relation is known from a previous result in the literature ${ }^{31}$ as

$$
\omega \frac{D}{2} \Im \oint_{\Omega}\left(W \frac{\partial}{\partial r} \Delta W^{*}-\Delta W^{*} \frac{\partial W}{\partial r}\right) R d \theta=0 .
$$

Applying this result to our work yields the following relationship which has to be fulfilled by the coefficients $\hat{A}_{q}$ and $\hat{B}_{q}$ if there is no absorption in the medium:

$$
\begin{gathered}
4 \omega D k_{p}^{2} \sum_{q}\left(\left|B_{q}^{(H)}\right|^{2}+\Re\left\{\left(A_{q}^{(J)}\right)^{*} B_{q}^{(H)}\right\}\right. \\
\left.+\frac{\pi}{2} \Im\left\{\left(A_{q}^{(I)}\right)^{*} B_{q}^{(K)}\right\}\right)=0 .
\end{gathered}
$$

Equation (27) was used to verify all of the numerical calculations. Convergence studies have been performed and values below $10^{-4}$ were obtained for all cases by setting the range of $q \in[-15,15]$.

\section{B. Commercial FEM simulator}

A commercial finite element simulator (COMSOL v4.4) has been used to calculate the behavior of the system. This simulator solves the full elastic equation (including longitudinal, shear vertical, and shear horizontal waves) to calculate the displacement field in a 3D model of the system. As incoming wave we have selected a plane wave $\left(\theta_{s}=0\right)$ at three values of the nondimensional frequency, $k_{p} R=[\pi, 2 \pi, 5 \pi]$. The system is also considered for three possible orientations of the internal beam: $\theta_{b}=\left[0^{\circ},-45^{\circ},-90^{\circ}\right]$, see Fig. 1 . The data is subsequently exported and compared visually against the analytical simulator coded using the theory described in Sec. IV.

The FEM simulator runs in an Intel Core i7-3930K @ $3.2 \mathrm{GHz}$ machine with $32 \mathrm{~GB}$ of RAM. Each simulation shown in Figs. 2 and 3 required 192.000 free tetrahedral elements to mesh the whole 3D volume and took approximately one hour to finish. The ones in Fig. 4 took $7 \mathrm{~h}$ to be completed due to the huge number of elements needed (over $1 \times 10^{6}$ ) to perform an accurate calculation.

Figure 2 shows the absolute value of the displacement obtained from the analytical simulator (left panels) and the FEM simulator (right panels) when a plane wave $\left(\theta_{s}=0\right)$ of non-dimensional frequency $k_{p} R=\pi$ impinges on the system. In a similar way, Fig. 3 and Fig. 4 show the results obtained from the analytical simulator (left panels) and the FEM simulator (right panels) for a non-dimensional frequency of $k_{p} R=2 \pi$ and $k_{p} R=5 \pi$, respectively. Notice that the scattering pattern created by the two simulators are very similar for all combinations of frequency and beam angle considered. Note particularly, the case when the beam is tilted at $\theta_{b}=-45^{\circ}$, where the discontinuities of the non-symmetric scattering pattern have been perfectly reproduced by the analytical simulator. It is evident from the three simulations that 

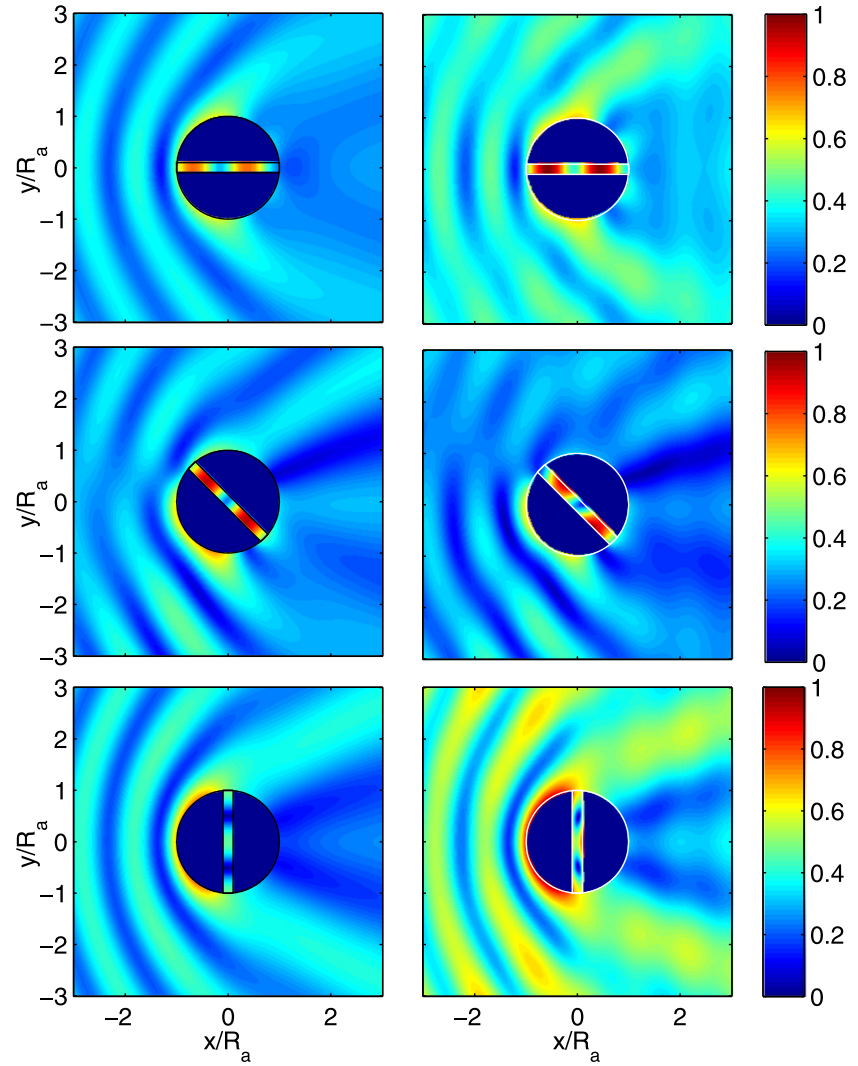

FIG. 2. (Color online) Absolute value of the displacement field produced when a plane wave $\left(\theta_{s}=0\right)$ with a non-dimensional frequency $k_{p} R=\pi$ impinges on the system for three different beam orientations $\theta_{b}=\left[0^{\circ},-45^{\circ},-90^{\circ}\right]$. The left column panels shows the results obtained by the analytical simulator and the right column panels show the ones obtained from the commercial finite element simulator.

the flexural wavelength in the internal beam are the same in both simulations, although the FEM simulator shows some 2D wave effects within the beam that are not modeled by the $1 \mathrm{D}$ beam theory.

From the comparison shown in Figs. 2-4 we can conclude that our semi-analytical modeling is stable and accurate in a broad band of frequencies, at least until $k_{p} R=5 \pi$, which is the maximum value considered here. We note that there are clearly some restrictions to our model: (i) it has the same limitations implicit in the Kirchhoff-Love and Euler-Bernoulli theories, which have been employed in the modeling; and (ii) the present analysis only applies to objects with axial symmetry. Restriction (i) could be relaxed using higher order plate and beam theories, although the analysis and matrix algebra will be more complicated. Regarding (ii), more general shapes could be considered following the methods described in Ref. 34.

\section{Far-field}

The amplitude of the far-field flexural waves is calculated as ${ }^{31}$

$$
\sigma_{s c}\left(k_{p}, \theta\right)=\left|\sqrt{\frac{2}{\pi k_{p}}} \sum_{q}(-i)^{q} B_{q}^{(H)} e^{i q \theta}\right| .
$$
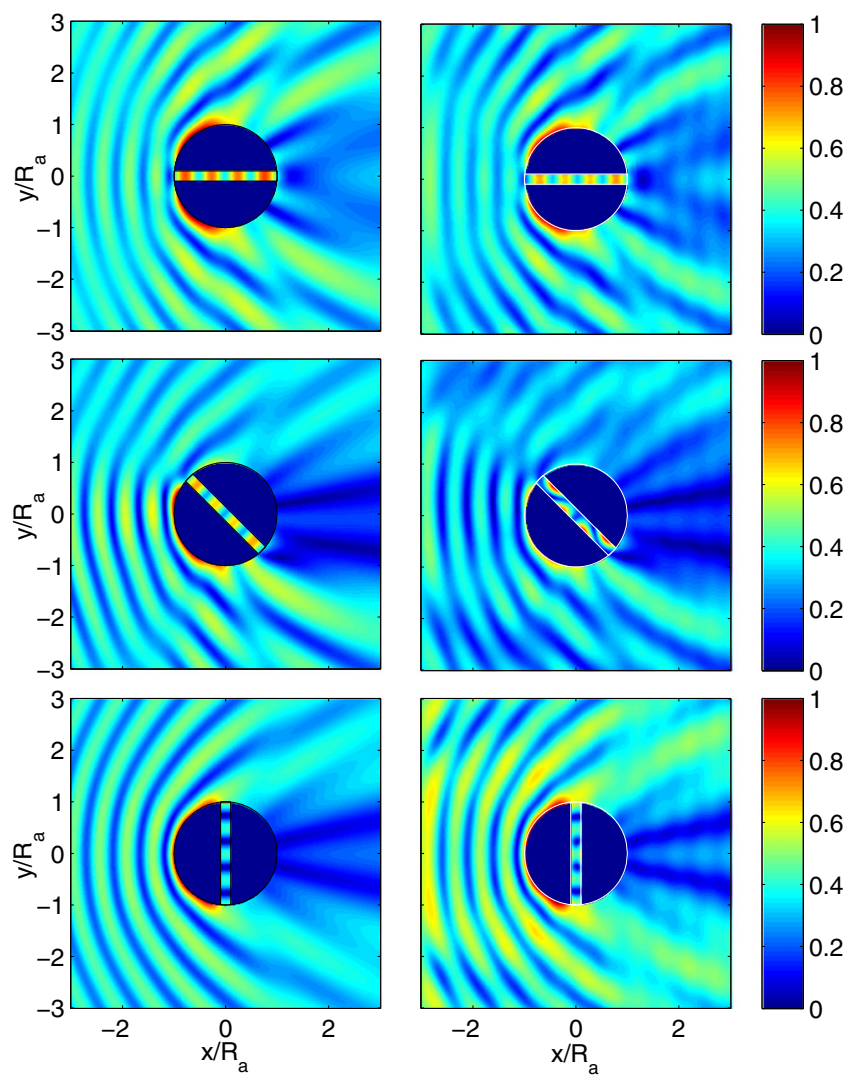

FIG. 3. (Color online) The same as for Fig. 2 except that the nondimensional frequency is $k_{p} R=2 \pi$.
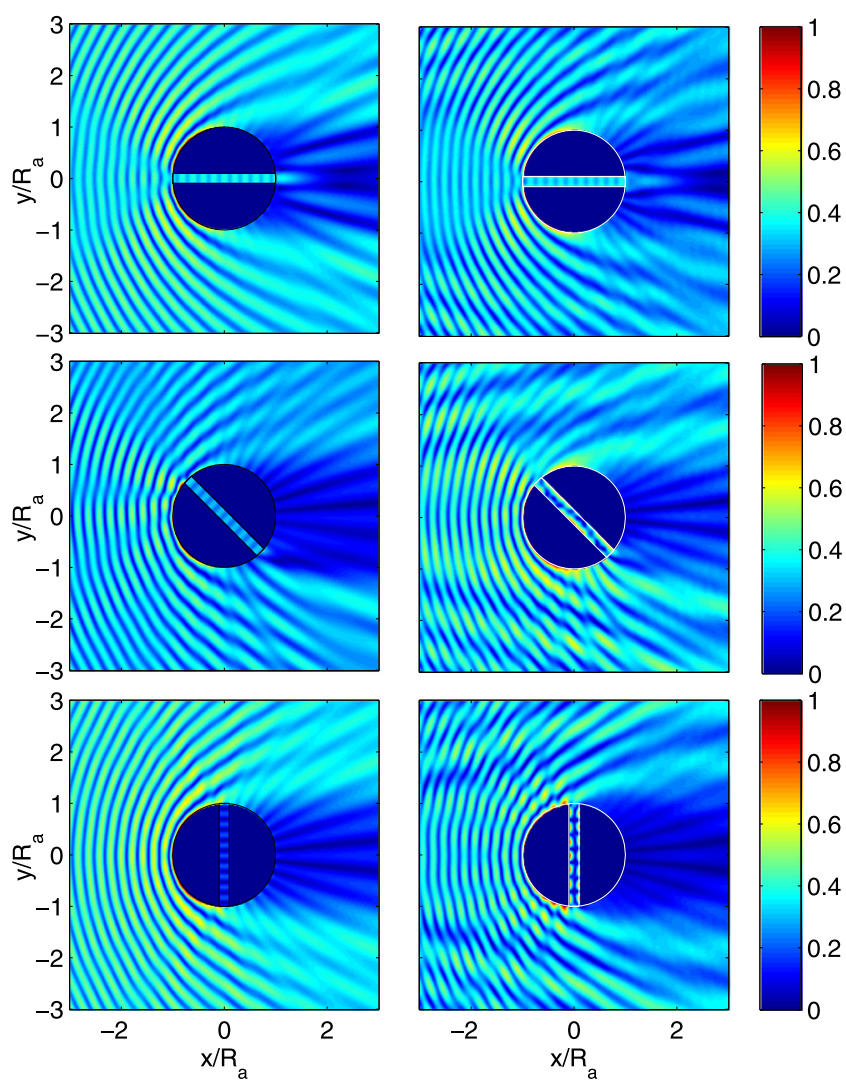

FIG. 4. (Color online) The same as for Fig. 2 except that the nondimensional frequency is $k_{p} R=5 \pi$. 

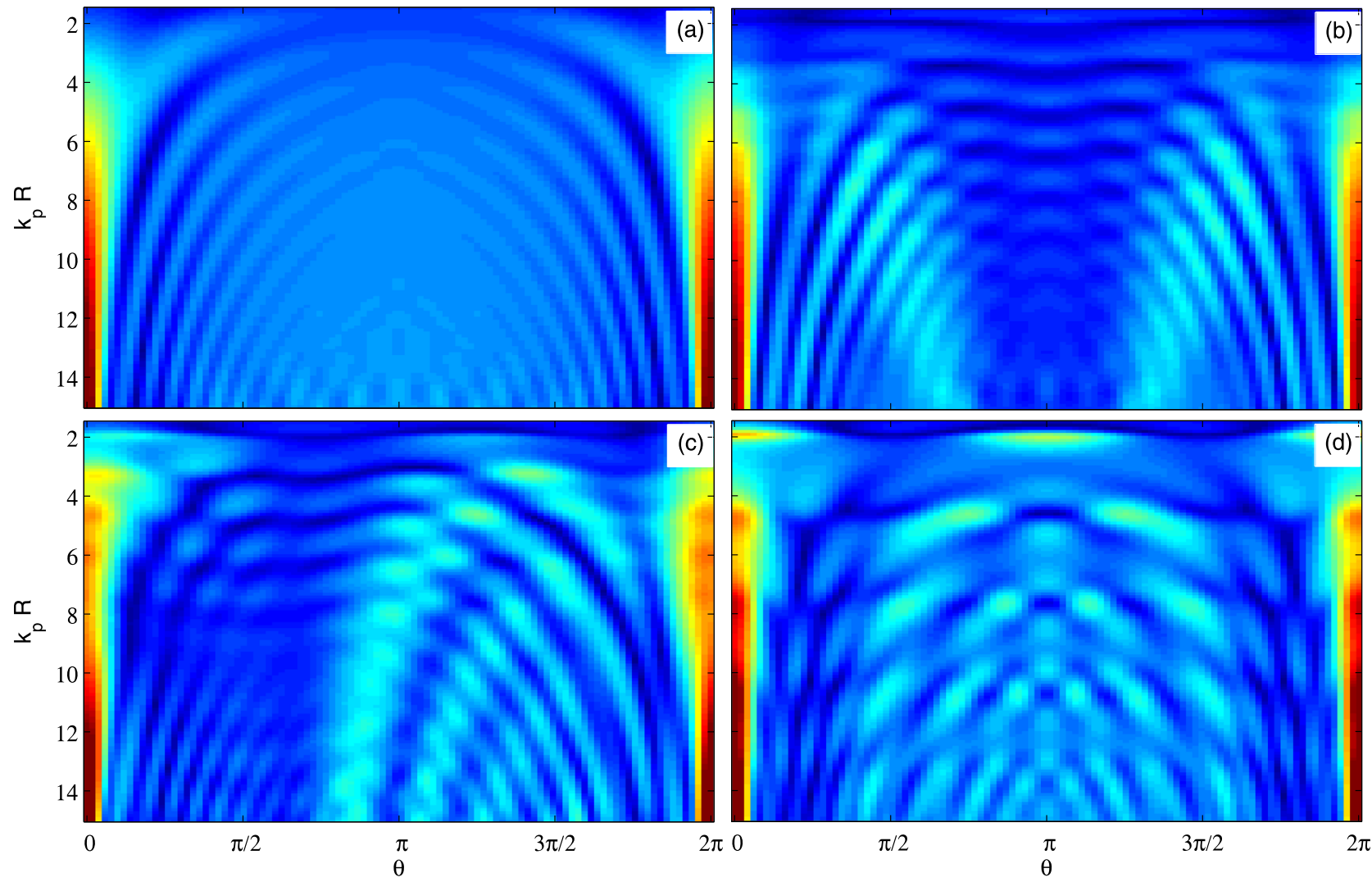

FIG. 5. (Color online) Far-field produced when a plane wave $\left(\theta_{s}=0\right)$ with non-dimensional frequencies ranging from $k_{p} R=0.5 \pi$ to $k_{p} R=5 \pi$ impinges a hole in a plate (a) and a hole with a crossing beam at three different orientations: $\theta_{b}=0^{\circ}$ (b), $\theta_{b}=-45^{\circ}(\mathrm{c})$, and $\theta_{b}=-90^{\circ}$ (d).

Figure 5 show the far-field produced when a plane wave $\left(\theta_{s}=0\right)$ with non-dimensional frequencies ranging from $k_{p} R=0.5 \pi$ to $k_{p} R=5 \pi$ impinges on the system. Figure 5(a) is the far-field produced by a hole in the plate. Notice that for the beam oriented at $\theta_{b}=0^{\circ}$ [Fig. 5(b)] the far-field around $\theta=\pi$ is lower than on Fig. 5(a) because the wave can enter the beam easily and propagate across it. On the other hand, in the position at $\theta_{b}=-90^{\circ}$ [Fig. 5(d)], the wave encounters a hole and so it is reflected producing more back-scattering. In the case of the position at $\theta_{b}=-45^{\circ}$ [Fig. 5(c)] there less back-scattering in $[0, \pi]$ than in $[\pi, 2 \pi]$ because the entrance to the beam is located at $\theta_{b}+\pi=3 \pi / 4$.

\section{CONCLUSIONS}

In this work, the impedance method for obtaining the T-matrix for flexural waves in a plate has been described. As an example of this method, the T-matrix of a thin plate with a hole traversed by a beam was solved. The procedure explicitly separates the Kirchhoff-Love and the Euler-Bernoulli solutions in the plate and beam and couples them in a manner that can be easily generalized to other theories and configurations. Additionally, an analytical simulator has been coded using the theory described in this work and the conservation of energy principle has been used for verification. Moreover, the results have been also tested against a commercial finite element simulator. Finally, the far-field behavior of the system has been presented for a wide bandwidth and different beam orientations, indicating a strong variability in the scattered field depending on the relative alignment of the beam with the incident wave.

\section{ACKNOWLEDGMENTS}

The work of A.C. and J.S.D. was supported by the U.S. Office of Naval Research (ONR) under Grant No. N000141210216 and the Spanish MINECO under project TEC 2010-19751 and that of A.N.N. was supported under ONR MURI Grant No. N000141310631. The authors acknowledge Alexey Titovich for helpful discussion.

\section{APPENDIX A: BOUNDARY CONDITIONS}

In this section, we explain how to obtain the boundary conditions for the system at hand. In the whole extent of this work, both displacements are positive in the positive $\hat{z}$-axis. Also, a positive slope is represented as an inclined black line. The moments in a 3D figure are represented using the double-headed arrow notation and the right-hand grip rule. In the case of a 2D figure, $\otimes$ and $\odot$ represent an ingoing and outgoing double arrow, respectively. Finally, the shear stresses are defined with a single-headed arrow.

Figure 6 shows all the moments and shear stresses defined for the Kirchhoff-Love plate theory. Notice that $M_{i j}^{\prime}$ $=M_{i j}+\left(\partial M_{i j} / \partial x_{i}\right) d x_{i}$ and $Q_{i j}^{\prime}=Q_{i j}+\left(\partial Q_{i j} / \partial x_{i}\right) d x_{i}$.

In a similar way, Fig. 7 displays the direction of the moments and shear stresses defined for the Euler-Bernoulli beam theory. Notice that $M_{x}^{\prime}=M_{x}+\left(\partial M_{x} / \partial x\right) d x$ and $Q_{x}^{\prime}=Q_{x}+\left(\partial Q_{x} / \partial x\right) d x$.

Finally, Fig. 8 shows the plate and the beam together. $\Psi_{1}$ is the right anchor point at $(r, \theta)=(R, 0)$ and $(x)=(+R)$; and $\Psi_{2}$ is the left anchor point at $(r, \theta)=(R, \pi)$ and 


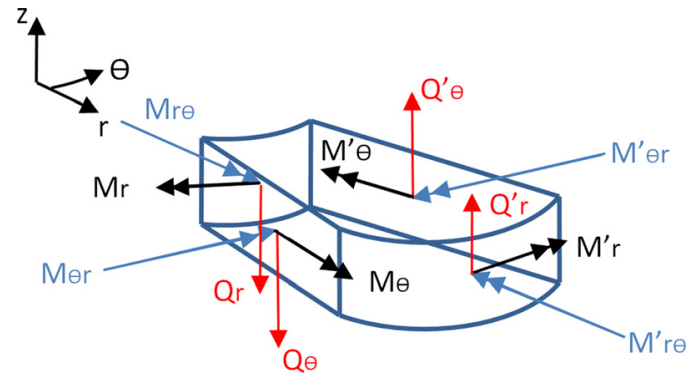

FIG. 6. (Color online) Scheme of the direction of all the moments and shear stresses defined by the Kirchhoff-Love plate theory. Notice that $M_{i j}^{\prime}$ $=M_{i j}+\left(\partial M_{i j} / \partial x_{i}\right) d x_{i}$ and $Q_{i j}^{\prime}=Q_{i j}+\left(\partial Q_{i j} / \partial x_{i}\right) d x_{i}$.

$(x)=(-R)$. The upper panel shows the displacements and the slopes and the lower panel shows the moments and the shear stresses. Notice that the Kirchhoff stress is defined as $V_{r}$ $=Q_{r}-(1 / r) \partial M_{r \theta} / \partial \theta$ so its direction is the same as $Q_{r}$.

The boundary conditions require that at the anchor points $\Psi_{i}$ the displacement and the slope are continuous and the sum of the moments and the sum of the stresses are zero. Looking at the two panels from Fig. 8 it is easy to obtain them as

$$
\begin{aligned}
& W(R, 0)=V(R) \text { and } W(R, \pi)=V(-R), \\
& \left.\frac{\partial W}{\partial r}\right|_{\substack{r=R \\
\theta=0}}=\left.\frac{\partial V}{\partial x}\right|_{x=R} \text { and }\left.\frac{\partial W}{\partial r}\right|_{\substack{r=R \\
\theta=\pi}}=-\left.\frac{\partial V}{\partial x}\right|_{x=-R}, \\
& \left.M_{r}\right|_{\substack{r=R \\
\theta=0}}=\left.\frac{M_{x}}{R}\right|_{x=R} \text { and }\left.\quad M_{r}\right|_{\substack{r=R \\
\theta=\pi}}=\left.\frac{M_{x}}{R}\right|_{x=-R}, \\
& \left.V_{r}\right|_{\substack{r=R \\
\theta=0}}=\left.\frac{Q_{x}}{R}\right|_{x=R} \text { and }\left.V_{r}\right|_{\substack{r=R \\
\theta=\pi}}=-\left.\frac{Q_{x}}{R}\right|_{x=-R} .
\end{aligned}
$$

Notice that the moments and the shear stresses of the beam are divided by the radius $R$ of the hole. It is useful to take into account the dimension of each term. The moment of the plate, $M_{r}$, has dimensions of $[N]$ while the moment of the beam, $M_{x}$, has dimensions of $[\mathrm{Nm}]$. At the same time, the Kirchhoff stress, $V_{r}$, has dimension of $[N / m]$ and the shear stress, $Q_{x}$, has dimensions of $[N]$. Therefore, if we integrate over a differential element $\varepsilon$,

$$
\begin{aligned}
& \int_{0-\epsilon}^{0+\epsilon} M_{r}(\theta) R d \theta=M_{x}, \\
& \int_{0-\epsilon}^{0+\epsilon} V_{r}(\theta) R d \theta=Q_{x},
\end{aligned}
$$

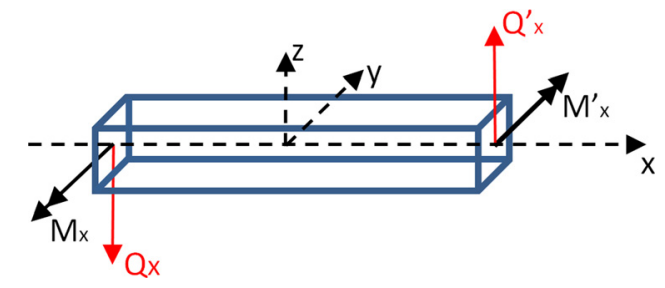

FIG. 7. (Color online) Scheme of the direction of all the moments and shear stresses defined by the Euler-Bernoulli beam theory. Notice that $M_{x}^{\prime}$ $=M_{x}+\left(\partial M_{x} / \partial x\right) d x$ and $Q_{x}^{\prime}=Q_{x}+\left(\partial Q_{x} / \partial x\right) d x$.

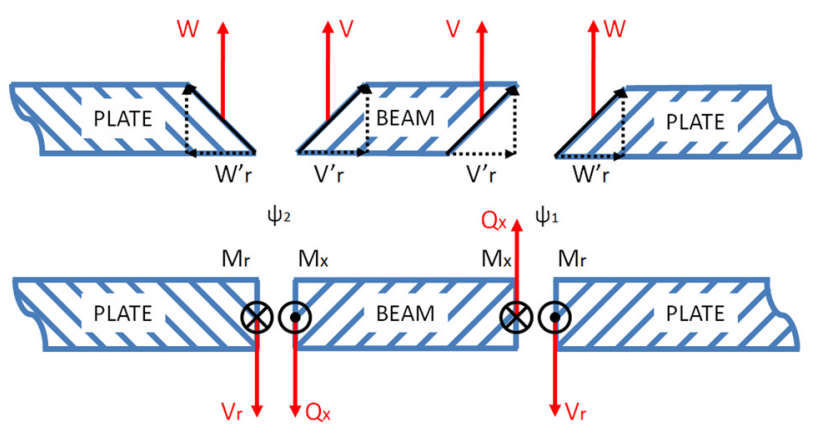

FIG. 8. (Color online) Scheme of the boundary conditions, displacements $(W, V)$, slopes $\left(W_{r}^{\prime}, V_{r}^{\prime}\right)$, moments $\left(M_{r}, M_{x}\right)$ and shear stresses $\left(V_{r}, Q_{x}\right)$, between the plate and the beam at the two anchor points $\Psi_{1}$ and $\Psi_{2}$.

we see that an additional term $1 / R$ has to be added to the moments and the shear stresses of the beam.

\section{APPENDIX B: IMPEDANCE METHOD}

The T-matrix is defined as $\hat{B}=\mathbf{T} \hat{A}$, relating the incident wave amplitudes to the scattered amplitudes. Using Einstein notation it becomes $\hat{B}_{q}=\mathbf{T}_{q s} \hat{A}_{s}$, where

$$
\hat{A}_{s}=\left[\begin{array}{c}
A_{s}^{(J)} \\
A_{s}^{(I)}
\end{array}\right] ; \quad \hat{B}_{q}=\left[\begin{array}{c}
B_{q}^{(H)} \\
B_{q}^{(K)}
\end{array}\right] ; \quad \mathbf{T}_{q s}=\left[\begin{array}{ll}
T_{11} & T_{12} \\
T_{21} & T_{22}
\end{array}\right] .
$$

In Ref. 33, the impedance matrices were defined by the relation between the pressure $P$ and the particle velocity $v$ at the boundary $\Omega$. Notice, that the boundary condition for a fluid-rigid interface is $v=0$ and for a fluid-vacuum interface it is $P=0$. In the case of a thin plate, the boundary conditions for a plate-clamped interface are $W=W_{r}^{\prime}=0$ and for a plate-fluid interface they are $M_{r}=V_{r}=0$. By analogy, we can define a new set of impedance matrices as

$$
\begin{aligned}
& {\left[\begin{array}{c}
M_{r} \\
V_{r}
\end{array}\right]_{q}^{(\text {inc })}=-\mathbf{Z}_{q s}^{(\text {inc })}\left[\begin{array}{c}
W \\
W_{r}^{\prime}
\end{array}\right]_{s}^{(\text {inc })} \text { at } \Omega,} \\
& {\left[\begin{array}{c}
M_{r} \\
V_{r}
\end{array}\right]_{q}^{(\text {scat })}=\mathbf{Z}_{q s}^{(\text {scat })}\left[\begin{array}{c}
W \\
W_{r}^{\prime}
\end{array}\right]_{s}^{(\text {scat })} \text { at } \Omega,} \\
& \left(\left[\begin{array}{c}
M_{r} \\
V_{r}
\end{array}\right]_{q}^{\text {(inc) }}+\left[\begin{array}{c}
M_{r} \\
V_{r}
\end{array}\right]_{q}^{(\text {scat })}\right. \\
& =-\mathbf{Z}_{q s}\left(\left[\begin{array}{c}
W \\
W_{r}^{\prime}
\end{array}\right]_{s}^{(\text {inc })}+\left[\begin{array}{c}
W \\
W_{r}^{\prime}
\end{array}\right]_{s}^{(\text {scat })}\right) \text { at } \Omega,
\end{aligned}
$$

where $\mathbf{Z}_{q s}^{\text {inc }}, \mathbf{Z}_{q s}^{\text {scat }}$, and $\mathbf{Z}_{q s}$ are the impedances for the incoming, scattered and internal wave, respectively. Notice that they are defined at the boundary $\Omega$. Solving for Equation (B2) we obtain the following relationship:

$$
\left[\begin{array}{c}
W \\
W_{r}^{\prime}
\end{array}\right]_{q}^{(\text {scat })}=\tilde{\mathbf{T}}_{q s}\left[\begin{array}{c}
W \\
W_{r}^{\prime}
\end{array}\right]_{s}^{(\text {inc })}
$$

where 


$$
\tilde{\mathbf{T}}_{q s}=\left[\mathbf{Z}_{q s}^{\text {scat }}+\mathbf{Z}_{q s}\right]^{-1}\left[\mathbf{Z}_{q s}^{\mathrm{inc}}-\mathbf{Z}_{q s}\right]
$$

As can be seen, $\tilde{\mathbf{T}}$ relates the displacement and the slope of the incoming and scattered wave at the boundary between the two media. In the case of a cylinder in a plate, these are given by

$$
\begin{aligned}
& {\left[\begin{array}{c}
W \\
W_{r}^{\prime}
\end{array}\right]_{q}^{(\mathrm{inc})}=\left[\begin{array}{cc}
J_{q}\left(k_{p} R\right) & I_{q}\left(k_{p} R\right) \\
k_{p} J_{q}^{\prime}\left(k_{p} R\right) & k_{p} I_{q}^{\prime}\left(k_{p} R\right)
\end{array}\right]\left[\begin{array}{c}
A_{q}^{(J)} \\
A_{q}^{(I)}
\end{array}\right] e^{i q \theta}=\left[\begin{array}{c}
\tilde{A}_{q}^{(J)} \\
\tilde{A}_{q}^{(I)}
\end{array}\right] e^{i q \theta},} \\
& {\left[\begin{array}{c}
W \\
W_{r}^{\prime}
\end{array}\right]_{q}^{(\text {scat })}=\left[\begin{array}{cc}
H_{q}\left(k_{p} R\right) & K_{q}\left(k_{p} R\right) \\
k_{p} H_{q}^{\prime}\left(k_{p} R\right) & k_{p} K_{q}^{\prime}\left(k_{p} R\right)
\end{array}\right]\left[\begin{array}{c}
B_{q}^{(H)} \\
B_{q}^{(K)}
\end{array}\right] e^{i q \theta}=\left[\begin{array}{c}
\tilde{B}_{q}^{J} \\
\tilde{B}_{q}^{(I)}
\end{array}\right] e^{i q \theta} .}
\end{aligned}
$$

Notice that $\tilde{B}=\tilde{\mathbf{T}} \tilde{A}$, from which it is easy to obtain the matrix $\mathbf{T}$ as a function of $\tilde{\mathbf{T}}$,

$$
\mathbf{T}_{q s}=\left[\mathbf{M}_{q q}^{H K}\left(k_{p}\right)\right]^{-1} \quad\left[\mathbf{Z}_{q s}^{\text {scat }}+\mathbf{Z}_{q s}\right]^{-1}\left[\mathbf{Z}_{q s}^{\text {inc }}-\mathbf{Z}_{q s}\right] \quad\left[\mathbf{M}_{s s}^{J I}\left(k_{p}\right)\right]
$$

where $\mathbf{M}$ is a quasi-diagonal matrix defined as

$$
\mathbf{M}_{q q}^{\Upsilon \Phi}\left(k_{i}\right)=\left[\begin{array}{cc}
\Upsilon_{q}\left(k_{i} R\right) & \Phi_{q}\left(k_{i} R\right) \\
k_{i} \Upsilon_{q}^{\prime}\left(k_{i} R\right) & k_{i} \Phi_{q}^{\prime}\left(k_{i} R\right)
\end{array}\right] .
$$

\section{APPENDIX C: BEAM STIFFNESS MATRIX}

From the definition of the displacement of the beam [Eq. (5)], we can write the following matrix:

$$
\left[\begin{array}{c}
V(x) \\
V^{\prime}(x) \\
V^{\prime \prime}(x) \\
V^{\prime \prime \prime}(x)
\end{array}\right]=\left[\begin{array}{cccc}
\left(i k_{b}\right)^{0} e^{i k_{b} x} & \left(-i k_{b}\right)^{0} e^{-i k_{b} x} & \left(k_{b}\right)^{0} e^{k_{b} x} & \left(-k_{b}\right)^{0} e^{-k_{b} x} \\
\left(i k_{b}\right)^{1} e^{i k_{b} x} & \left(-i k_{b}\right)^{1} e^{-i k_{b} x} & \left(k_{b}\right)^{1} e^{k_{b} x} & \left(-k_{b}\right)^{1} e^{-k_{b} x} \\
\left(i k_{b}\right)^{2} e^{i k_{b} x} & \left(-i k_{b}\right)^{2} e^{-i k_{b} x} & \left(k_{b}\right)^{2} e^{k_{b} x} & \left(-k_{b}\right)^{2} e^{-k_{b} x} \\
\left(i k_{b}\right)^{3} e^{i k_{b} x} & \left(-i k_{b}\right)^{3} e^{-i k_{b} x} & \left(k_{b}\right)^{3} e^{k_{b} x} & \left(-k_{b}\right)^{3} e^{-k_{b} x}
\end{array}\right]\left[\begin{array}{c}
C^{I} \\
C^{I I} \\
D^{I} \\
D^{I I}
\end{array}\right]
$$

Evaluating this at the end points $x= \pm R$, simplifying and ordering terms we obtain

$$
\begin{aligned}
& {\left[\begin{array}{c}
V(-R) \\
V^{\prime}(-R) \\
V(R) \\
V^{\prime}(R)
\end{array}\right]=\left[\begin{array}{ccrr}
e^{-i k_{b} R} & e^{i k_{b} R} & e^{-k_{b} R} & e^{k_{b} R} \\
i k_{b} e^{-i k_{b} R} & -i k_{b} e^{i k_{b} R} & k_{b} e^{-k_{b} R} & -k_{b} e^{k_{b} R} \\
e^{i k_{b} R} & e^{-i k_{b} R} & e^{k_{b} R} & e^{-k_{b} R} \\
i k_{b} e^{i k_{b} R} & -i k_{b} e^{-i k_{b} R} & k_{b} e^{k_{b} R} & -k_{b} e^{-k_{b} R}
\end{array}\right]\left[\begin{array}{c}
C^{I} \\
C^{I I} \\
D^{I} \\
D^{I I}
\end{array}\right]=\mathbf{L}_{1}\left[\begin{array}{c}
C^{I} \\
C^{I I} \\
D^{I} \\
D^{I I}
\end{array}\right],} \\
& {\left[\begin{array}{c}
V^{\prime \prime}(-R) \\
V^{\prime \prime \prime}(-R) \\
V^{\prime \prime}(R) \\
V^{\prime \prime \prime}(R)
\end{array}\right]=k_{b}^{2}\left[\begin{array}{cccc}
-e^{-i k_{b} R} & -e^{i k_{b} R} & e^{-k_{b} R} & e^{k_{b} R} \\
-i k_{b} e^{-i k_{b} R} & i k_{b} e^{i k_{b} R} & k_{b} e^{-k_{b} R} & -k_{b} e^{k_{b} R} \\
-e^{i k_{b} R} & -e^{-i k_{b} R} & e^{k_{b} R} & e^{-k_{b} R} \\
-i k_{b} e^{i k_{b} R} & i k_{b} e^{-i k_{b} R} & k_{b} e^{k_{b} R} & -k_{b} e^{-k_{b} R}
\end{array}\right]\left[\begin{array}{c}
C^{I} \\
C^{I I} \\
D^{I} \\
D^{I I}
\end{array}\right]=k_{b}^{2} \mathbf{L}_{2}\left[\begin{array}{c}
C^{I} \\
C^{I I} \\
D^{I} \\
D^{I I}
\end{array}\right] .}
\end{aligned}
$$

Taking into account the required boundary conditions [Eqs. (7)], the sign of some rows has to change. Therefore,

$$
\mathbf{L}_{1}^{\prime}=\left[\begin{array}{llll}
1 & & & \\
& -1 & & \\
& & 1 & \\
& & & 1
\end{array}\right] \mathbf{L}_{1} \quad ; \quad \mathbf{L}_{2}^{\prime}=\left[\begin{array}{llll}
1 & & & \\
& -1 & & \\
& & 1 & \\
& & & 1
\end{array}\right] \mathbf{L}_{2} \text {. }
$$

By combining the three previous equations, we obtain the moment and the shear stress as a function of the displacement and the slope as 


$$
\left[\begin{array}{c}
M_{-R} \\
-Q_{-R} \\
M_{R} \\
Q_{R}
\end{array}\right]=-E I k_{b}^{2}\left[\mathbf{L}_{2}^{\prime}\right]\left[\mathbf{L}_{1}^{\prime}\right]^{-1}\left[\begin{array}{c}
V_{-R} \\
-V_{-R}^{\prime} \\
V_{R} \\
V_{R}^{\prime}
\end{array}\right]=\mathbf{K}\left[\begin{array}{c}
V_{-R} \\
-V_{-R}^{\prime} \\
V_{R} \\
V_{R}^{\prime}
\end{array}\right]
$$

where the stiffness matrix $\mathbf{K}$ is simplified and has the following final form:

$$
\mathbf{K}=\frac{-E I k_{b}^{2}}{1-c c_{h}}\left[\begin{array}{cccc}
-s s_{h} & k_{b}^{-1}\left(s c_{h}-c s_{h}\right) & c_{h}-c & -k_{b}^{-1}\left(s_{h}-s\right) \\
-k_{b}\left(c s_{h}+s c_{h}\right) & s s_{h} & k_{b}\left(s+s_{h}\right) & c-c_{h} \\
c_{h}-c & -k_{b}^{-1}\left(s_{h}-s\right) & -s s_{h} & k_{b}^{-1}\left(s c_{h}-c s_{h}\right) \\
k_{b}\left(s+s_{h}\right) & c-c_{h} & -k_{b}\left(c s_{h}+s c_{h}\right) & s s_{h}
\end{array}\right]
$$

with $c=\cos \left(2 k_{b} R\right), s=\sin \left(2 k_{b} R\right), c_{h}=\cosh \left(2 k_{b} R\right)$, and $s_{h}=\sinh \left(2 k_{b} R\right)$.

${ }^{1}$ J.-C. Hsu and T.-T. Wu, "Efficient formulation for band-structure calculations of two-dimensional phononic-crystal plates," Phys. Rev. B 74, 144303 (2006).

${ }^{2}$ R. C. McPhedran, A. B. Movchan, and N. V. Movchan, "Platonic crystals: Bloch bands, neutrality and defects," Mech. Mater. 41, 356-363 (2009).

${ }^{3}$ T.-T. Wu, Y.-T. Chen, J.-H. Sun, S.-C. S. Lin, and T. J. Huang, "Focusing of the lowest antisymmetric Lamb wave in a gradient-index phononic crystal plate," Appl. Phys. Lett. 98, 171911 (2011).

${ }^{4}$ M. Farhat, S. Guenneau, and S. Enoch, "High directivity and confinement of flexural waves through ultra-refraction in thin perforated plates," Europhys. Lett. 91, 54003 (2010).

${ }^{5}$ J. Pierre, O. Boyko, L. Belliard, J. O. Vasseur, and B. Bonello, "Negative refraction of zero order flexural Lamb waves through a two-dimensional phononic crystal,” Appl. Phys. Lett. 97, 121919 (2010).

${ }^{6}$ M. Farhat, S. Guenneau, S. Enoch, A. B. Movchan, and G. G. Petursson, "Focusing bending waves via negative refraction in perforated thin plates,” Appl. Phys. Lett. 96, 081909 (2010).

${ }^{7}$ S. Bramhavar, C. Prada, A. A. Maznev, A. G. Every, T. B. Norris, and T. W. Murray, "Negative refraction and focusing of elastic Lamb waves at an interface,” Phys. Rev. B 83, 014106 (2011).

${ }^{8}$ A. N. Norris and A. L. Shuvalov, "Elastic cloaking theory," Wave Motion 48, 525-538 (2011).

${ }^{9}$ N. Stenger, M. Wilhelm, and M. Wegener, "Experiments on elastic cloaking in thin plates," Phys. Rev. Lett. 108, 014301 (2012).

${ }^{10}$ V. V. Krylov and F. J. B. S. Tilman, "Acoustic black holes for flexural waves as effective vibration dampers," J. Sound. Vib. 274, 605-619 (2004).

${ }^{11}$ V. V. Krylov and R. Winward, "Experimental investigation of the acoustic black hole effect for flexural waves in tapered plates," J. Sound. Vib. 300, 43-49 (2007).

${ }^{12}$ V. V. Krylov, "Acoustic black holes and their applications for vibration damping and sound absorption," in Proceedings of the International Conference on Noise and Vibration Engineering (ISMA 2012), pp. 933-944.

${ }^{13}$ A. Climente, D. Torrent, and J. Sánchez-Dehesa, "Omnidirectional broadband insulating device for flexural waves in thin plates," J. Appl. Phys. 114, 214903 (2013).

${ }^{14}$ D. Yu, Y. Liu, H. Zhao, G. Wang, and J. Qiu, "Flexural vibration band gaps in Euler-Bernoulli beams with locally resonant structures with two degrees of freedom," Phys. Rev. B 73, 064301 (2006).

${ }^{15}$ D. Yu, Y. Liu, G. Wang, H. Zhao, and J. Qiu, "Flexural vibration band gaps in Timoshenko beams with locally resonant structures," J. Appl. Phys. 100, 124901 (2006).

${ }^{16}$ A. Khelif, Y. Achaoui, S. Benchabane, V. Laude, and B. Aoubiza, "Locally resonant surface acoustic wave band gaps in a two-dimensional phononic crystal of pillars on a surface," Phys. Rev. B 81, 214303 (2010).
${ }^{17}$ R. Sainidou, B. Djafari-Rouhani, Y. Pennec, and J. O. Vasseur, "Locally resonant phononic crystals made of hollow spheres or cylinders," Phys. Rev. B 73, 024302 (2006).

${ }^{18}$ Y. Xiao, B. R. Mace, J. Wen, and X. Wen, "Formation and coupling of band gaps in a locally resonant elastic system comprising a string with attached resonators," Phys. Lett. A 375, 1485-1491 (2011).

${ }^{19}$ Y. Xiao, J. Wen, and X. Wen, "Flexural wave band gaps in locally resonant thin plates with periodically attached springmass resonators," J. Phys. D: Appl. Phys. 45, 195401 (2012).

${ }^{20}$ Y. Pennec, B. Djafari-Rouhani, H. Larabi, J. O. Vasseur, and A. C. Hladky-Hennion, "Low-frequency gaps in a phononic crystal constituted of cylindrical dots deposited on a thin homogeneous plate," Phys. Rev. B 78, 104105 (2008).

${ }^{21}$ M. Oudich, Y. Li, B. M. Assouar, and Z. Hou, "A sonic band gap based on the locally resonant phononic plates with stubs,” New J. Phys. 12, 083049 (2010).

${ }^{22}$ T.-T. Wu, Z.-G. Huang, T.-C. Tsai, and T.-C. Wu, "Evidence of complete band gap and resonances in a plate with periodic stubbed surface," Appl. Phys. Lett. 93, 111902 (2008).

${ }^{23}$ M. Oudich, M. Senesi, M. B. Assouar, M. Ruzenne, J.-H. Sun, B. Vincent, Z. Hou, and T.-T. Wu, "Experimental evidence of locally resonant sonic band gap in two-dimensional phononic stubbed plates," Phys. Rev. B 84, 165136 (2011).

${ }^{24}$ F. Casadei, M. Ruzzene, L. Dozio, and K. A. Cunefare, "Broadband vibration control through periodic arrays of resonant shunts: Experimental investigation on plates," Smart Mater. Struct. 19, 015002 (2010).

${ }^{25}$ M. Badreddine Assouar, M. Senesi, M. Oudich, M. Ruzzene, and Z. Hou, "Broadband plate-type acoustic metamaterial for low-frequency sound attenuation," Appl. Phys. Lett. 101, 173505 (2012).

${ }^{26}$ D. Torrent, D. Mayou, and J. Sanchez-Dehesa, "Elastic analog of graphene: Dirac cones and edge states for flexural waves in thin plates," Phys. Rev. B 87, 115143 (2013).

${ }^{27}$ T.-T. Wu, J.-C. Hsu, and J.-H. Sun, "Phononic plate waves," IEEE Trans. Ultrason. Ferroelectr. Freq. Control 58, 2146-2161 (2011).

${ }^{28}$ M. I. Hussein, M. J. Leamy, and M. Ruzzene, "Dynamics of phononic materials and structures: Historical origins, recent progress, and future outlook,” Appl. Mech. Rev. 66, 040802 (2014).

${ }^{29} \mathrm{~B}$. Mace, "Discussion: 'Dynamics of phononic materials and structures: Historical origins, recent progress and future outlook' (Hussein, M. I., Leamy, M. J., and Ruzzene, M.),” Appl. Mech. Rev. 66, 045502 (2014).

${ }^{30} \mathrm{E}$. Andreassen, K. Manktelow, and M. Ruzzene, "Directional bending wave propagation in periodically perforated plates," J. Sound. Vib. 335, 187-203 (2015).

${ }^{31}$ A. N. Norris and C. Vemula, "Scattering of flexural waves on thin plates," J. Sound. Vib. 181, 115-125 (1995).

${ }^{32}$ V. V. Matus and V. F. Emets, "T-matrix method formulation applied to the study of flexural waves scattering from a through obstacle in a plate," J. Sound. Vib. 329, 2843-2850 (2010).

${ }^{33}$ Y. Bobrovnitskii, "Impedance theory of sound scattering: General relations," Acoust. Phys. 52, 513-517 (2006).

${ }^{34}$ A. S. Titovich and A. N. Norris, "Tunable cylindrical shell as an element in acoustic metamaterial," J. Acoust. Soc. Am. 136, 1601-1609 (2014). 